\title{
Integrating Buprenorphine Treatment Into Family Medicine Resident Clinic
}

\author{
Jeffrey D. Tiemstra, MD; Lauren H. Walsh, MD, MPH
}

\begin{abstract}
BACKGROUND AND OBJECTIVES: Medication-assisted treatment (MAT) for opioid use disorder with buprenorphine in primary care is effective and patient-accessible yet remains underutilized, including among residency training programs. One concern in residency programs is that MAT patients must be seen at least monthly and will overwhelm residents' clinic schedules and dilute their clinical experience. Our family medicine residency initiated an MAT program integrated into residents' continuity clinic schedules. After 2 years we assessed the chronic medical comorbidities we were managing in our MAT population.
\end{abstract}

METHODS: We performed a retrospective review of all active patients receiving MAT. We collected basic demographic data and whether we were the patient's primary care provider (PCP) or were only providing MAT. For the patients for whom we were the PCP we recorded the chronic comorbidities that required medical management.

RESULTS: One hundred fifty-seven active patients were $52 \%$ male and $48 \%$ female. The mean age was 38 years $(S D=10)$ with a range of 22 to 77 years, with nine patients over age 60 years $(6 \%)$. One hundred three patients used us as their PCP (66\%). For these patients the mean number of chronic comorbidities was 2.3; only 10 patients reported no comorbidities. Psychiatric comorbidities were the most common with $69 \%$ of patients with a mood disorder, although nonpsychiatric comorbidities still averaged 1.5 per patient.

CONCLUSIONS: MAT integrated into family medicine resident continuity clinics provides a broad and substantial primary care clinical experience for residents.

(Fam Med. 2020;52(9):653-5.)

doi: 10.22454/FamMed.2020.783547

$\mathrm{n}$ response to the nationwide opioid epidemic, medication-assisted treatment (MAT) with buprenorphine has been made available to primary care practices with a relatively modest investment of time and resources. MAT is highly effective and promises greater availability to patients through primary care. ${ }^{1}$ Unfortunately training programs have been slow to respond to this opportunity. A 2017 study of family medicine residencies reported only $29 \%$ had a required addiction medicine curriculum, and only $36 \%$ had a faculty member with a buprenorphine waiver. ${ }^{2}$ Similarly a 2018 survey of family medicine, internal medicine, and psychiatry programs found only $36 \%$ of programs included training in MAT and only $23 \%$ encouraged residents to obtain their waiver. ${ }^{3}$ This represents a critical missed opportunity, since MAT training in residency is strongly associated with providing it in practice. ${ }^{4}$

Due to requirements for at least monthly visits and regular drug testing MAT is often provided in dedicated settings. This structure segregates these patients to sites where comprehensive primary care will not be readily available, and data confirms that patients engaged in MAT receive less primary care than matched controls. ${ }^{5}$ Integrating MAT into comprehensive primary care is clearly needed.

In January 2018 we began providing MAT in our resident continuity clinic. In our rural county only one other physician offers MAT, so many patients travelled 50 miles or more for treatment. All clinic precepting faculty obtained their buprenorphine waiver. Initially we advertised the program to local physicians so our first patients were referrals who had a PCP, but after the first few months most patients were coming by word of mouth. Many had no PCP and so established with us.

From Aurora Health Care Rural Family Medicine Residency, Elkhorn, WI. 
All our residents see MAT patients, complete the 8-hour training course at the start of PGY2, and have their buprenorphine waiver by PGY3. MAT patients are scheduled throughout the regular clinic schedule and residents will see one or more almost daily.

Since MAT in our practice grew quickly we were concerned that it might impact residents' breadth of experience in their continuity practice. We decided to examine what comorbidities this population presented to our practice to assess the impact of the MAT program on residents' overall primary care experience.

\section{Methods}

We performed a retrospective descriptive study of our current MAT patient population to assess the range of primary care clinical experiences offered to residents by this population. We identified patients from our list of active MAT patients maintained in our elecronic health record (EHR). We identified MAT patients who had also chosen us as their PCP using the EHR PCP field, that we maintain with residents' names for their continuity patients. We record ourselves as PCP only when actively requested by the patient. We then reviewed these charts for the presence of chronic comorbidities in addition to opioid use disorder (OUD). We counted only active chronic conditions with ongoing physician management, identified by having a coded billing diagnosis on a visit with an assessment and plan. For psychiatric disorders we grouped mood disorders together since there was substantial overlap of patients with anxiety and depressive disorders. We counted bipolar disorders separately since these disorders can pose substantially greater challenges to management. We did not include visits for acute illnesses and injuries. We calculated percentages of patients with each diagnosis or category as well as per-patient averages for psychiatric and nonpsychiatric diagnoses. The Advocate Aurora Institutional Review Board approved this study.

\section{Results}

Of 157 active patients $52 \%$ were male and $48 \%$ female. The mean age was 38 years $(\mathrm{SD}=10)$ with a range of 22 to 77 years, with nine patients over age 60 years (6\%); 103 patients used our residents as their PCP $(66 \%)$. Comorbidities and their frequencies for these patients are shown in Table 1. The mean number of chronic comorbidities was 2.3, with only 10 patients with none. Psychiatric comorbidities were the most common, with $69 \%$ of patients with some form of mood disorder, $72 \%$ with any mental health disorder, and an average of 0.9 mental health conditions per patient. Nonpsychiatric comorbidities averaged 1.5 per patient. Chronic pain syndrome (musculoskeletal) was the most common at $44 \%$, including degenerative spine and joint disease, failed back surgery syndrome, other postoperative chronic pain, and fibromyalgia. Asthma, hypertension, gastroesophageal reflux, and hepatitis $\mathrm{C}$ all occurred with frequencies greater than $10 \%$, and the remaining comorbidities represent other common primary care problems.

\section{Discussion}

Patients with OUD are known to have increased risk for mental health disorders, hepatitis $\mathrm{C}$ and HIV infection. ${ }^{6}$ Older patients with OUD have been shown to have increased rates of hypertension, cardiovascular disease, and polypharmacy, ${ }^{7}$ and patients with OUD and diabetes mellitus have been shown to have more diabetic complications. ${ }^{8}$ Our literature review did not locate any data on comorbidities among MAT patients specifically in family medicine residencies. This data along with our results suggests there is both a need for primary care and an opportunity for resident clinical experience with this population. $\mathrm{Pa}$ tients with OUD lack primary care for many reasons; in our community many have been dismissed from other primary care practices or have financial and social stresses that have led them to neglect their overall health care.

Psychiatric comorbidities clearly represent a major challenge in this population. Few of our new patients are actively engaged in mental health care. A few will connect with psychiatry but in most cases we manage mental health issues completely or in cooperation with counseling services. In our rural area access to services is very limited with long wait times for all but the most urgent situations.

It is not surprising that this population, with its wide age and balanced gender distribution, would experience other comorbidities expected in the general population. Next steps include exploring how overall primary care is affected by having a visit agenda that includes MAT, and how resident-patient continuity is impacted. But residency programs considering MAT training and experience should be confident that including MAT as part of comprehensive primary care will offer the broad range of clinical experience desired for residents, as well as address unmet needs in the community. 
Table 1: Chronic Comorbidities Among 103 Resident Continuity Clinic Patients on MAT

\begin{tabular}{|c|c|c|}
\hline Psychiatric Comorbidities & $\mathbf{N}$ & Percent \\
\hline Mood disorders (not including bipolar disorders) & 45 & 44 \\
\hline Bipolar disorders & 26 & 25 \\
\hline Attention deficit disorder & 18 & 18 \\
\hline Any psychiatric disorder & 74 & 72 \\
\hline Nonpsychiatric Comorbidities & $\mathbf{N}$ & Percent \\
\hline Chronic pain syndrome (musculoskeletal, fibromyalgia, postsurgical) & 45 & 44 \\
\hline Asthma & 16 & 16 \\
\hline Hypertension & 15 & 15 \\
\hline Gastroesophageal reflux & 13 & 13 \\
\hline Hepatitis C & 12 & 12 \\
\hline Migraine & 8 & 8 \\
\hline Chronic obstructive pulmonary disease & 6 & 6 \\
\hline Seizure disorders & 6 & 6 \\
\hline Hypothyroidism & 6 & 6 \\
\hline Dysfunctional uterine bleeding & 5 & 5 \\
\hline Rheumatoid/psoriatic arthritis & 4 & 4 \\
\hline Diabetes mellitus & 3 & 3 \\
\hline Contraception & 3 & 3 \\
\hline Erectile dysfunction & 3 & 3 \\
\hline Anemia & 2 & 2 \\
\hline Acne & 2 & 2 \\
\hline Atrial fibrillation with cardiovascular disease & 1 & 1 \\
\hline None & 10 & 10 \\
\hline Totals & $\mathbf{N}$ & Mean/Patient \\
\hline Psychiatric comorbidities & 89 & 0.86 \\
\hline Nonpsychiatric comorbidities & 150 & 1.46 \\
\hline All comorbidities & 239 & 2.32 \\
\hline
\end{tabular}

Abbreviation: MAT, medication-assisted treatment.

CORRESPONDING AUTHOR: Address correspondence to Dr Jeffrey D. Tiemstra, Aurora Lakeland Family Medicine Clinic, W3985 County Road NN, Elkhorn, WI 53121. 262-741-2121. Fax: 262-741-2175. Jeffrey.Tiemstra@aah.org.

\section{References}

1. Oesterle TS, Thusius NJ, Rummans TA, Gold MS. Medication-assisted treatment for opioiduse disorder. Mayo Clin Proc. 2019;94(10):20722086.

2. Tong S, Sabo R, Aycock R, et al. Assessment of addiction medicine training in family medicine residency programs: a CERA study. Fam Med. 2017;49(7):537-543.
3. Tesema L, Marshall J, Hathaway R, et al Training in office-based opioid treatment with buprenorphine in US residency programs: A national survey of residency program directors. Subst Abus. 2018;39(4):434-440.

4. Tong ST, Hochheimer CJ, Peterson LE, Krist AH. Buprenorphine provision by early career family physicians. Ann Fam Med 2018;16(5):443-446

5. Spithoff S, Kiran T, Khuu W, et al. Quality of primary care among individuals receiving treatment for opioid use disorder. Can Fam Physician. 2019;65(5):343-351.
6. Pearce LA, Min JE, Piske M, et al. Opioid agonist treatment and risk of mortality during opioid overdose public health emergency: population based retrospective cohort study. BMJ. 2020;368:m772.

7. Oh G, Abner EL, Fardo DW, Freeman PR, Moga DC. Patterns and predictors of chronic opioid use in older adults: a retrospective cohort study. PLoS One. 2019;14(1):e0210341.

8. Winhusen T, Theobald J, Kaelber DC, Lewis D. Medical complications associated with substance use disorders in patients with type 2 diabetes and hypertension: electronic health record findings. Addiction. 2019;114(8):14621470. 